Article

\title{
Collaborating with Management Academics in a New Economy: Benefits and Challenges
}

\author{
Yongyan $\mathrm{Li}^{1, *}$ (1) and Guangwei $\mathrm{Hu}^{2}$ \\ 1 Faculty of Education, University of Hong Kong, Hong Kong, China \\ 2 Department of English, Hong Kong Polytechnic University, Hong Kong, China; guangwei.hu@polyu.edu.hk \\ * Correspondence: yongyan@hku.hk; Tel.: +852-3917-2415
}

Received: 6 December 2017; Accepted: 8 January 2018; Published: 10 January 2018

\begin{abstract}
As a response to intensified globalization, international research collaboration has become common in the social sciences. This paper reports a study that examined what Chinese management academics and their overseas counterparts perceived to be the benefits and challenges arising from research collaboration with each other. Data collected with two parallel questionnaires administered, respectively, to 114 Chinese and 30 overseas management academics revealed a variety of perceived benefits relating mainly to Chinese and overseas academics' complementing strengths. Analysis of the same data also identified an array of perceived challenges stemming from a combination of cultural, epistemological, ideological, linguistic, institutional, and relational differences. Our study generated insights to be drawn upon in policy-making and in the coordination of international research collaboration.
\end{abstract}

Keywords: international research collaboration; social sciences; scholarly publication

\section{Introduction}

International research collaboration has been an important feature of academia across disciplines. In this paper, we focus on such collaboration in the discipline of management in the context of mainland China. As management research has a broad disciplinary scope [1], we should clarify at the outset that we are specifically concerned with the disciplinary field of business and organizational management, with this focus aligned with the scope of interest of the International Association for Chinese Management Research (IACMR) (see also [2,3]). It is significant to focus on the field of business and organizational management in a study of international research collaboration, as collaborative scholarship is common in this field and has far-reaching practical implications in the globalization of commercial activities [4].

Previous research on Chinese academics' international collaboration was almost exclusively based on bibliometric methods (e.g., [1,5]). What bibliometric research falls short of, as Melkers and Kiopa [6] (p. 408) have pointed out, is the social dynamics behind the collaboration (see also Hoffman et al., 2014). In this paper we report a study based on questionnaire data which helped to reveal such social dynamics. Our study was aimed to uncover what Chinese management academics (CMAs) and overseas management academics (OMAs) would perceive to be the benefits of and challenges in engaging in research collaboration with each other. In our definition, CMAs are those affiliated to mainland Chinese universities, while OMAs are mostly affiliated to universities outside of mainland China (either Chinese-ethnic or non-Chinese-ethnic), with a small number being expatriate academics employed in mainland Chinese universities. The findings of the study will have implications for researchers, administrators, as well as policy-makers in terms of fostering fair, equal, productive, and mutually benefiting research collaboration. 


\section{International Research Collaboration and an "Asymmetrical Structure of Exchange" in Social Sciences}

International research collaboration, typically leading to co-publications involving authors of different nationalities, has been a fast-growing feature of scientific research and continues to intensify in both natural and social sciences, so that increasingly knowledge production is shared and issues of global relevance are tackled [7]. International collaboration provides access to resources that complement one's own stock [8-11] with respect to data, research skills, funding, equipment, human resources, and language skills [2,12-14]. Benefits of international collaboration, such as enhancing research performance and output, boosting citation statistics, and broadening research horizons, have been widely acknowledged $[6,12,15,16]$. On the other hand, the challenges of cross-border research collaboration are also well recognized and may include cost in time and resources, inconvenience in communication caused by physical distance (even though the Internet has been a major facilitator of communication), conflicting aims, differing institutional systems, issue of trust, power relations, linguistic and ideological boundaries, and ontological and epistemological differences across cultures [14,17-19].

When compared with the natural sciences, some of these challenges (i.e., disagreements in ideology, ontology, and epistemology) would be more manifest in research collaboration in the social sciences, which "are more connected to and imbedded in (and thus affected by) the social and political system to which they are oriented" [20] (p. 594) and [21]. The same reason may have also reinforced "a very strong core-periphery pattern" and "a highly asymmetrical structure of exchange" in international collaboration in the social sciences [7] (p. 144), with North America and Western Europe continuing to dominate the dynamics of knowledge production. In this "core-periphery", "asymmetrical" environment, the mounting pressure for periphery academics to publish in English-medium indexed journals [22] would prompt their "assimilation" to the center-dominant paradigm [23] (p. 299), and encourage their engagement in international collaboration as a valuable strategy [24].

In China, international academic collaboration has historically been emphasized by the government with funding support and installment of academic exchange programs [1]. In the past two decades, amidst a wide-spread internationalization drive at Chinese universities, international collaboration has been further stimulated through various policy-level initiatives, for example, rewarding publication in international, indexed journals handsomely [25], luring back overseas-trained academics [26], and focusing on collaborating with high-performers (i.e., the United States (U.S.) and the European Union (EU) nations) [27]. Bibliometric research has shown that the proportion of the internationally indexed articles co-authored by Chinese and overseas academics has been on the rise in both natural and social sciences over the decades $[5,20]$.

\section{Chinese Social Sciences "Moving towards the World" and CMAs' International Research Collaboration}

China's reform and opening policy in 1978 ushered in a new era for social sciences in the country, with the ensuing course of development manifested as a process of Westernization, characterized by the worshiping of Western theories and methods amongst Chinese social scientists. For an increasing number of Chinese scholars, however, Westernization has "subjugated Chinese social sciences to Western cultural hegemony" and "served to reduce the academic autonomy of Chinese social sciences" [28] (p. 183). In reaction to Westernization, a movement of indigenization arose, emphasizing the need to understand the strengths and limitations of Western theories and methods [29] and calling for the cultivation of a sense of locality to set up research agendas that address local societal needs and build theories rooted in the Chinese context. For more recent advocates along the line, such a sense of locality nurtures academic autonomy in Chinese social sciences and forms the basis of their "Moving towards the world" [28] (p. 183). 
While the debates over how to move towards the world are still going on, a consensus amongst Chinese social scientists by and large seemed to be: in moving towards the world they should combine the goals of learning from Western scientific research methodology, and of achieving local relevance-that is, eliminating the dichotomy between scientificization and localization (e.g., [30]). For the younger generation of Chinese social scientists, in particular, especially those who are overseas-trained or have had overseas stints during or subsequent to doctoral study (i.e., the academics who are on the frontiers of international publication), scientificization of scholarship based on mastery of rigorous research methodology embodied in contemporary Western social science research, as well as familiarity with existing Western theories (studied through a critical lens) is of paramount importance for Chinese social sciences to move towards the world. The case of the discipline of management illustrates the viewpoint particularly well. The International Association for Chinese Management Research (IACMR), since its establishment in 2002 under the leadership of some Chinese OMAs, in particular, Anne S. Tsui (Chinese American), former president of the U.S.-based Academy of Management, has been running seminars and research methodology workshops in major cities regularly, with well-established OMAs or (overseas-trained) CMAs serving as the speakers, and attracting attendees from universities all over the country. Eminently, while working to rectify "the lack of advanced training in scientific methods" [4] (p. 5), amongst CMAs in general, IACMR also emphasized the criterion of local relevance, committed to a mission of building "Chinese theories of management" $[4,31]$, "through serious engagement in deep contextualization, novel questioning, and innovative theorizing" [32] (p. 1353).

The collaboration between CMAs and OMAs has been a cornerstone of IACMR's pledged mission in China. Through the training sessions on research methodology, its publishing of English and Chinese journals, biennial international conferences, and other initiatives, IACMR has created "excellent platforms for scholarly conversations on Chinese management research" [31] (p. 25) between CMAs and OMAs. Tsui [32] (p. 1360) spoke of the importance of "seasoned senior scholars working in partnership with international novices", pointing out that "Such intercultural and intergenerational collaboration may be a pragmatic approach to realizing their joint aspiration of making a difference through significant scholarship".

Through its varied, sustained initiatives, IACMR has taken CMAs on a path of situated learning through peripheral participation [33], in relation to the global community of management research. OMAs, on the other hand, through participating in IACMR's initiatives, are supporting CMAs' as well as their own situated learning, for the engagement leads them to new phenomena in the Chinese context and helps them develop indigenous perspectives to understand complex cultural-historical situations and overcome U.S.-centrism in research topics and methods [17,24]. (After all, according to Lave and Wenger [33], all members of a community of practice, varying in their level of expertise, are engaged in peripheral participation in the community.) At the same time, however, challenges as outlined in the previous section for international collaboration are likely to exist between CMAs and OMAs. Research is needed to gain insights into the two sides' perspectives on both the benefits to be gained from their collaboration and the challenges that would hinder it.

\section{Methods}

Our study thus was guided by this research question: How do CMAs and OMAs perceive the benefits of and challenges in engaging in research collaboration with each other? To access our potential participants for the study, we availed ourselves on the occasion of IACMR's 6th biennial conference held in June 2014 in Beijing (see also [3]). As the webpage of the conference showed a Participant List with names of the delegates and their affiliations, we were able to retrieve the email addresses of most of the delegates and following the conclusion of the conference, we sent to each target participant an email containing a URL link to a questionnaire mounted on SurveyMonkey ${ }^{\circledR}$. CMAs received Questionnaire One, which was written in Chinese and focused on their use of English for academic communication; OMAs received Questionnaire Two, which was written in English and 
centered on their international research collaboration with CMAs. We gathered a total of 114 valid responses to Questionnaire One (response rate 28.9\%) and 30 valid responses to Questionnaire Two (response rate 12.1\%). The data that formed the basis of the study reported in this paper were mainly derived from two open-ended questions in Questionnaire One that asked CMAs, respectively, about the perceived benefits of and challenges in collaborating with OMAs, and three open-ended questions in Questionnaire Two that elicited OMAs' perceptions of benefits of and challenges in collaborating with CMAs, and possible changes in the pattern of research collaboration with CMAs (see Appendix A for these open-ended questions in Questionnaire One and Questionnaire Two respectively).

The respondents' answers to the open-ended questions were loaded into NVivo, a qualitative data analysis software program, and analyzed through descriptive coding [34], where it was the wording of the participants, rather than researchers' interpretation of meaning, which was relied on during the process of analysis. Our hierarchy of codes had a relatively straightforward structure: "CMAs on benefits of collaboration", "CMAs on challenges of collaboration", "OMAs on benefits of collaboration", and "OMAs on challenges of collaboration" were used as the higher-level codes. The various perceived benefits and challenges, as summarized in Tables 1 and 2 in the Results section, were subsumed under the four higher-level codes respectively. Each lower-level code, as a category of benefit or challenge from the perspective of CMAs or OMAs, further subsumed a list of codes derived directly from the data, which were mostly in vivo codes [35] representing the exact wording of the participants.

\section{Results}

If joint authorship between OMAs and CMAs is taken to signal international collaboration, the conference program of IACMR2014 provided a glimpse of the extent of such collaboration. Of all the 259 English papers and 167 Chinese papers presented at the conference, 56 (21.6\%) and 8 (4.8\%), respectively, were coauthored by CMAs and OMAs. These formed a minority of the presentations delivered at the conference. If this pattern was characteristic of collaboration on other projects, we may thus suggest that of the 114 CMAs who responded to Questionnaire One, only some would have had the actual experience of collaborating with OMAs. One CMA respondent noted: "I have never collaborated with overseas academics; this perception comes mainly from my experience at IACMR". As we aimed to obtain the respondents' perspectives and views, rather than reflections upon actual experience, we consider the observations made by those without experience of collaboration a valuable part of our data. Of the 30 OMA respondents to Questionnaire Two, in responding to a background information question, no one chose the option of "I do not have any mainland Chinese collaborators", indicating that all 30 respondents had collaborative relationships with CMAs, which may have motivated their responding to our questionnaire. It is further worth noting that when asked about the purposes of their visits to China in the past five years, 22 (73.3\%) out of 30 selected the option of "To meet with Chinese collaborators to discuss projects".

In the following, we will present findings to address our research question. The quotations from our CMA respondents are our translations from Chinese, while those from the OMA respondents are in English in the original.

\subsection{CMAs' Perspectives on Benefits of Collaborating with OMAs}

Table 1 summarizes CMAs' perspectives on collaborating with OMAs, with the number of mentions for each point indicated in brackets.

Table 1 shows that broadening horizons and gaining other perspectives and raising the quality of academic research topped the list of benefits named by our CMA respondents, together accounting for $38.9 \%$ of the total 157 comments on benefits. Some extracts for the first category are:

"To broaden horizons, maintain academic vigor and impact"

"To understand the research question from different perspectives and angles" 
"International horizon"

"To learn of the frontiers of the discipline"

"Overseas scholars are well informed of the research frontiers"

"To connect track with international academia in research"

"To learn about new research methods and ways of thinking in research"

"More comprehensive viewpoints"

"Diversified perspectives, more frontier viewpoints on research topics"

Table 1. Chinese management academics (CMAs) $(n=114)$ on benefits of and challenges in collaborating with overseas management academics (OMAs).

\begin{tabular}{cc}
\hline \multicolumn{1}{c}{ Benefits } & Challenges \\
\hline $\begin{array}{c}\text { Broadening horizons and gaining other perspectives } \\
(31)\end{array}$ & $\begin{array}{c}\text { Difficulty in communication caused by language barriers } \\
\text { and geographic distance (49) }\end{array}$ \\
\hline Raising the quality of academic research (30) & $\begin{array}{c}\text { Different understandings of research issues, and different } \\
\text { perspectives and views on what is important (20) }\end{array}$ \\
\hline Getting help in English (25) & Coordination of pace and schedule (15) \\
\hline Learning the ropes of English academic writing (23) & Lacking access to potential collaborators (13) \\
\hline Facilitating publication in international journals (18) & Difficulty created by gaps in research competencies (7) \\
\hline Complementing each other academically (15) & Potential conflicts over authorship, responsibilities, \\
and gains (7)
\end{tabular}

A majority of comments that fall into the category of raising the quality of academic research bear upon the perceived more advanced research paradigm and more rigorous methodology on the part of overseas researchers and CMAs' passion in learning from their overseas counterparts in this regard. Some examples of their comments include:

"The scientific research methods"

"Systematic methods"

"Overseas academics' research methods are more rigorous"

"The standardization of research paradigms"

"To learn the latest research methods and tools"

"To enhance research standards"

Getting help in English and learning the ropes of English academic writing are two other sets of benefits nominated by the CMAs with high frequencies. CMAs were unambiguous about the benefit derived from collaborating with counterparts with stronger English abilities for co-authoring English papers:

"To overcome language barrier"

"Fluency in English"

"To obtain assistance with English language"

"To revise and polish language"

"Revisions of language"

"To polish English"

Learning the ropes of English academic writing, felt strongly by CMAs as a benefit of collaborating with OMAs, was suggested many times:

"Different paradigms of research writing in Chinese and English" 
"English writing and organization of the articles"

"(To produce) standardized English writing"

"(To learn) the paradigm of research writing"

"To learn about the ways English articles are written"

"How to compose a high-quality paper (including the formulation of the initial ideas, research design, research plan, and research writing)"

"Native English-speaking academics can provide help in writing"

"More standardized and rigorous research thinking and academic writing"

Facilitating publication in international journals, which is closely bound to the categories exemplified above, was acknowledged by the CMAs:

"(To ensure) the English articles written can be understood by reviewers"

"Channels to get published"

"Easier to publish articles"

"They (OMAs) know the journals well"

"Understanding of leading journals' standards"

"(Achieve) academic standardization, and publish high-quality papers"

"(Make it possible to publish in) top journals"

"To publish high-quality papers efficiently"

Gaining access to theoretical developments was also prominent for CMAs in international research collaboration:

"Overseas academics use more advanced theories"

"To share (with us) the latest research theories"

"To enrich and improve the frameworks and theories of research"

"(Overseas academics) have a solid basis in theories"

"Theoretical deduction and summarization of contribution"

"To decide on research topics and content, mainly in terms of theoretical framework, instead of the phenomenon to investigate"

The last point, in particular, highlights the formulation of "theoretical framework" as a main challenge for CMAs, when they are in no shortage of topics of study, and hence research data.

If in naming the above range of benefits CMAs projected themselves more in the role of a learner in need of broadening their horizons, getting English help, learning advanced research methods and English writing conventions, as well as gaining access to theories, some CMA respondents did acknowledge complementing each other academically as a benefit of international collaboration, thus projecting a more equal status between themselves and OMAs:

"To learn from each other's academic thoughts"

"To complement each other"

"To benefit from each other's advantages"

"Exchange of ideas"

"Strong-strong cooperation"

"(Collaboration) helps promote innovations in the research area"

Finally, it is worth noting that there were two mentions of OMAs' good qualities by our CMA respondents as benefits of collaboration:

"Intellectual passion, professionalism"

"They are reliable"

As will be seen later, our OMA respondents also held in high regard what they saw as CMAs' good qualities. 


\subsection{CMAs' Perspectives on Challenges in Collaborating with OMAs}

As shown in Table 1, our CMA respondents also named a range of challenges that might exist in research collaboration with overseas counterparts. The most often mentioned challenges concerned difficulty in communication caused by language barriers and geographic distance, accounting for $42.2 \%$ of the total 116 comments on challenges. Some examples of comments in the category are as follows:

"Cannot meet up very often, which causes inconvenience in communication and discussion"

"Distance in space makes discussions inconvenient"

"Oftentimes long-distance communication is needed, and it is not easy to do that frequently"

"Communication is not smooth"

$44.9 \%$ of the 49 comments in the category explicitly addressed the English language barrier, a focus that echoed CMAs' perceptions of getting help in English as a benefit, illustrated above. Thus, we had:

"A lack of self-confidence in face-to-face communication due to the barrier of oral English"

"My English communication skills (speaking and writing) are rather poor"

"Mainly language barrier"

"Problems in English writing and communication"

"If your English level is too low, collaboration can be difficult"

"If the academics are non-Chinese, communication can be difficult"

Another difficulty in communication was perceived to be caused by different understandings of research issues, and different perspectives and views on what is important:

"Ways of thinking"

"(Different) values"

"Cross cultural communication is not easy sometimes"

"Interested in different issues"

"Differences in cultural backgrounds, especially in the social sciences research"

"To persuade them that the phenomenon under study is significant in China, and to make them interested"

"Theoretical model/contribution and the practical meaning to Chinese management"

These comments point to potential divergence in the research issues that may be of interest and practical relevance for CMAs and OMAs; at a deeper level, they may also reflect cross-cultural differences in ideology, ontology, and epistemology (e.g., [17-19]).

The category of coordination of pace and schedule as a challenge has the following manifestations:

"Control of the pace of work"

"Synchronization in research"

"Progress is too slow"

"Time schedule can be delayed"

"Both sides are busy"

"They (OMAs) are very busy"

Apparently, the coordination of progress is also linked to the difficulty in communication caused by geographic distance as noted above. Nearly an equal number of CMAs put down lacking access to potential collaborators as another challenge:

"A lack of channels to communicate and collaborate"

"A lack of related support and channels"

"Network" 
"Cannot find ideal collaborators"

"It is hard to find collaborators who share the same interests and pursuits (with you)"

"The funding and time needed for overseas academic visits and attending conferences"

"Very few people can put you into contact with such collaborators"

"Lacking a platform to communicate with overseas scholars from related areas"

On the lack of "a platform" as noted in the last example shown above, the same respondent went on to note: "After all, for the majority of domestic researchers, opportunities to attend prestigious conferences (e.g., AOM in the U.S.) and to communicate with them are rare". Clearly the IACMR biennial conferences are meant to be such a platform for CMAs to communicate with their overseas peers; yet it is likely that for many CMAs, other than the existence of a potential "platform", brokers who can mediate such communication, or "put you into contact with such collaborators", can play a crucial role.

Difficulty created by gaps in research competencies echoes in particular to the benefit of raising the quality of academic research illustrated earlier. Such gaps may cause difficulty in finding collaborators or in engaging in collaboration, from the point of view of CMAs:

"Domestic management study lags far behind that of the overseas countries/research, it is hard to find collaborators that suit you and satisfy your needs"

"Demand on your own academic skills is high"

"My own level is not up to the standard"

"If your own level is rather low, it is hard to collaborate on an equal footing"

"Not familiar with overseas scholars; my own level is low"

"I am willing, but it is beyond my competency"

Some CMA respondents referred to potential conflicts over authorship, responsibilities, and gains, with the following remarks:

"Regarding authorship"

"Once there are collaborators involved, my own place (in the authorship byline) will be changed-something you can hardly object to"

"How to define responsibilities and rights of the two parties"

"Unbalanced contributions made to the paper"

"Overseas academics benefit more"

"Overseas scholars invest very little; usually it is us who compose the first draft. Such collaboration can hardly be deepened or maintained for long, we can learn very little from it, and the progress is slow"

As will be seen later, some OMA respondents expressed disapproval over their Chinese collaborators' views of authorship. The differing views over authorship and definition of responsibilities, and concerns over one's own interest constitute a potential source of conflict between the collaborating parties.

Finally, CMAs also viewed achieving mutual understanding and trust as a challenging process:

"Concerns over research results being published elsewhere"

"A lack of understanding and trust"

"Mutual understanding"

"Need to know each other well, to collaborate on the basis of mutual trust"

Mutual understanding and trust need to be built through communication; difficulty in communication caused by language barriers and geographic distance, as illustrated earlier, clearly would undermine the achievement of the goal. 


\subsection{OMAs' Perspectives on Benefits of Collaborating with CMAs}

Table 2 summarizes OMAs' perspectives on the benefits of collaborating with CMAs, with the number of mentions for each category likewise indicated in brackets.

Table 2. OMAs $(n=30)$ on benefits of and challenges in collaborating with CMAs.

\begin{tabular}{cc}
\hline Benefits & Challenges \\
\hline Access to local data and resources (25) & CMAs' training-related inadequacies (12) \\
\hline Gaining indigenous perspectives (9) & $\begin{array}{c}\text { Difficulty in communication caused by geographic } \\
\text { distance, and coordination of pace and schedule (8) }\end{array}$ \\
\hline CMAs' good qualities (5) & $\begin{array}{c}\text { Potential conflicts over authorship and } \\
\text { responsibilities (6) }\end{array}$ \\
\hline Helping with the training of CMAs (4) & $\begin{array}{c}\text { CMAs' inadequacies in English proficiency and } \\
\text { English writing ability (5) }\end{array}$ \\
\hline
\end{tabular}

Of the 30 OMA respondents, $25(83.3 \%)$ referred to access to local data and resources as a benefit of collaborating with CMAs, with such access covering data, access to local firms, and CMAs' data analysis skills and resources (the advantage of funding and having research students to help with research):

"Data collection"

"Access to companies and data"

"The variety of research data"

"Data, ideas, network with the industry"

"Benefited from data collection and analysis"

"(CMAs') data analysis skill"

"They are close to the data sources with more resources, e.g., doc students to collect data"

"Data collection and research funding"

Together with access to local data and resources, OMAs also tended to cite gaining indigenous perspectives as a benefit of collaborating with CMAs:

"China specific knowledge"

"Context knowledge"

"China is exciting"

"Helped me with research in emerging markets"

"Indigenous perspective, rich insight into Chinese culture and history, network with local firms and academics"

OMAs also praised CMAs' good qualities:

"Upbeat morale, motivation to publish"

"Diligence, good time management"

"Hard working"

Recalling CMAs' appreciation of OMAs' good qualities as shown earlier, we may see that mutual appreciation of attitudinal qualities is a plus in research collaboration.

Finally, a few OMA respondents saw research collaboration with CMAs as an opportunity for helping with the training of $C M A s$ :

"It is good to be able to help mainland colleagues"

"Help them learn"

"Nurture their development as a scholar" 
"HELP THEM TO GROW UP" (capital letters in the original)

These comments might appear to reflect a condescending stance on the part of the OMAs. Viewed in context and particularly in relation to OMAs' perspectives on CMAs' insufficient training as posing particular challenges to collaboration (see below), these observations were probably fair and the underlying mentoring intentions would be welcomed by CMAs.

\subsection{OMAs' Perspectives on Challenges in Collaborating with CMAs}

To OMAs, CMAs' training-related inadequacies constituted the leading challenge they perceived in research collaboration. The inadequacies were perceived to manifest themselves in managing the research process, engaging with the literature, formulating theoretical insights, observing ethical codes, and writing up research:

"Sometimes Chinese researchers do not understand the academic research purpose; sometimes they are unable to fulfill their roles"

"Lack of depth with literature"

"Research and writing capabilities"

"Ethics and quality of data"

"Lack of understanding of research process to meet IRB (Institutional Review Board) requirement"

"Unable to lead a project, lack theoretical insight"

"THEY DO NEED MORE TRAINING" (capital letters in the original)

Difficulty in communication caused by geographic distance, and coordination of pace and schedule, named by CMAs as challenges in international collaboration, were likewise noted by OMAs:

"Long distance"

"Lack of communication"

"Sometimes it is hard to control"

"Meeting deadlines"

"Time management"

"Their (CMAs') (other) commitments and constant distractions"

"Their (CMAs') busy work schedule"

As with CMAs, potential conflicts over authorship and responsibilities were also raised by OMAs, but in a light that was different from CMAs' perspectives illustrated earlier. Two related comments went:

"The mindset of authorship from mainland Chinese needs substantial improvement, at least from my experience. They may need (to) understand about what contribute to an authorship and what constitute intellectual contribution".

"Sometimes they (CMAs) did not show commitment; only care about SSCI publications, not on the areas of contributions".

Such comments can be seen as linked to OMAs' complaints of CMAs' insufficient training in the conduct of academic research as presented earlier. At the same time, it can be suggested that institutional pressures on CMAs to publish SSCI papers (see [36]) seem to be exerting a negative impact on international collaboration, potentially causing problems in commitment and tensions over authorship. Related potential conflicts may stem from:

"CMAs' way of thinking in doing research"

"Target journals are different"

"Cooperation and collaboration is tinged with competition"

The last point which views "competition" negatively may actually be interpreted in a positive light with credit to CMAs: that perhaps an increasing number of them aim to play an active role in 
collaboration—beyond being a mere "data collector" [2]—and to collaborate with overseas counterparts on an equal footing.

Finally, CMAs' inadequacies in English language proficiency and English writing ability were also pointed out:

"(CMAs' inadequate) writing capabilities"

"(CMAs' inadequate) language proficiency"

"(CMAs') proficiency in English writing remains to be improved"

"Language issues and (CMAs') difficulties in writing fluently"

This observation echoes CMAs' lament of challenges caused by the English language barrier, as well as their perceptions of the benefits in getting help in English and enhancing the mastery of English academic writing through collaboration.

\section{Discussion}

The foregoing section presented evidence to address our research question regarding CMAs' and OMAs' perceptions of benefits and challenges arising from research collaboration with each other. In terms of the perceived benefits, the two parties' perspectives can be explained by a resource-based view of collaboration [11], in that they saw complementation of their resources constituting the foundation of successful collaboration (e.g., [2,10,14]). Apart from explicitly referring to complementing each other academically as a benefit of collaborating with OMAs, our CMA respondents' perceptions of benefits of international collaboration above all rested on an appreciation of the opportunities afforded to learn from their overseas partners and improve themselves. They envisioned the benefits of accessing alternative perspectives [16] and emphasized the benefits of raising the quality of research by engaging with the criterion of rigor, learning the ropes of English academic writing, and gaining access to theoretical developments. It may be suggested that such perspectives displayed features of "academic dependency" [37]. Nevertheless, with the tendency of Westernization repudiated, such learning and borrowing apparently constitutes a needed force in Chinese social scientists' endeavor to achieve academic autonomy. Within the discipline of management, IACMR's decade-plus dedication to training CMAs in advanced research methods has been driven by the belief that Chinese management research, to make a valuable contribution to the global management knowledge base, should abide by "the criterion of rigor that all scientific studies are expected to meet" [4] (p. 6). Yet, probably for average CMAs, insufficiency in research training remains an issue, so that our OMA respondents' top concern in terms of challenges for collaboration was CMAs' training-related inadequacies. One gap in their Chinese counterparts' training was perceived by a few OMA respondents to lie in observing ethical codes in research (e.g., "Lack of understanding of research process to meet IRB requirement"). Although IRBs are increasingly set up in academic institutions in China [38], we believe that practicing high ethical standards in the research process remains a goal that Chinese social science researchers need to work for, and yet the current assessment culture that puts a premium on voluminous publishing threatens to erode this effort at the same time [39]. Nevertheless, the need to change the status quo is perhaps urgent, given the increasing contact that a global audience is making with the Chinese academics and Chinese universities. Initiatives should be taken at the institutional level to establish IRBs and create ethical clearance protocols. In this regard, research managers and policy makers at mainland Chinese universities can learn from the excellent examples long established at the universities in Hong Kong, where research ethics are safeguarded through rigorous ethics application procedures and self-monitoring that was set at high standards.

CMAs also valued receiving guidance on theories from OMAs. On this account, without subscribing to an "assimilation" approach to existing theories [23] (p. 299), it has been pointed out that theorizing based on contextualized research actually requires familiarity with extant theories developed in other contexts (e.g., in the Western center), to avoid "reinventing theories already in the literature" [31] (p. 24) and to be confident that the new theories developed are sensitive to the 
local context [4]. In gaining access to theoretical developments, CMAs should go beyond perfunctory borrowing. As having been suggested for the discipline of management [4] and for social sciences in China more generally [25], many Chinese researchers apply Western theories without "a native understanding of the borrowed theory" [40] (p. 50), which requires "a broad understanding of its historical roots, including the particular question it was intended to address, the pre-existing explanations, if any, it challenged, etc.". (p. 46). A similar issue exists in learning advanced research methods to raise the level of academic research, which was named by our CMA respondents as a high-priority benefit of international collaboration. Tsui [4] (p. 5) observed that "Chinese scholars are smart and quickly learn how to apply research methods, but this does not mean that they have necessarily understood the underlying epistemology or ontology". If ontological and epistemological differences across cultures pose challenges in cross-border collaboration [17,18], Tsui's observation suggests that such differences could stem from one party's failure to understand "the underlying epistemology or ontology" of the research methodology that both parties agree to adopt.

CMAs' keenness to enhance their mastery of English academic writing through participating in collaboration with OMAs was closely connected to another benefit they perceived of such collaboration: facilitating publication in international journals. There was thus an instrumental or pragmatic motive here [41]. However, placed in the context of Chinese scholars" lamenting of "non-normative practices" [29] (p. 71) in China's social studies and journal publications, as well as their call for the standardization of research and reporting practices, borrowing the standards of English academic writing (which is expected to embody the criterion of rigor of research) can have a positive impact on the presentation of research in the Chinese medium as well [42], apart from facilitating Chinese scholars' international publication effort.

In contrast to CMAs' perspectives, OMAs' observations of the gains of working with their Chinese counterparts foregrounded access to local data and resources and gaining indigenous perspectives-benefits of collaboration having long been acknowledged especially in the discipline of management $[14,17,24,43]$. The need to develop global management knowledge requires research being carried out in contexts other than the Western center to develop new theories; at the same time, there is also a need to find out whether the existing theories developed based on research conducted in the center apply in other contexts and how to enrich and refine the current theories [24,32,44]. While local knowledge rooted in the periphery may suffer from "parochialism" in the eyes of gatekeepers and be barred from publication in a center-based journal [22], research that is not informed by the world outside the center has been dubbed a "parochial dinosaur" in the context of management research [45]. International collaboration helps OMAs to overcome U.S.-centrism [24] and provides opportunities for them to jointly develop with CMAs grounded theories and locally-workable research methods [43].

Our OMA respondents also nominated helping with the training of CMAs as a benefit of collaborating with the latter. The seemingly condescending view can nevertheless be cast in a positive light. As suggested earlier in the paper, through the platform of exchange created by IACMR between OMAs and CMAs, for a growing number of OMAs, China has been both a vibrant and productive research base and a venue for participating in the training of Chinese colleagues. The collaborations between CMAs and OMAs, by facilitating situated learning [33] for both parties, are mediating the generation of "significant scholarship" envisioned by Tsui [32] (p. 1360). Yet, CMAs and OMAs may have different understandings of research issues, and different perspectives and views on what is important, according to our CMA respondents. The ideological orientations [19] of the two sides, the influence of contextual/local knowledge [14] on the part of CMAs, the two parties' training backgrounds and previous research experience, aims of research, target journals, and understandings of the expectations of the target journals, may all contribute to the rise of such challenges. Previous research (e.g., [21]) suggests that these challenges can be managed through the collaborating parties' personal and collective deliberations.

For both CMA and OMA respondents in our study, geographic distance could create difficulty in communication and obstruct coordination of pace and schedule [18]. The inconvenience brought by 
the lack of physical proximity can be mitigated to some extent by technology (e.g., communicating via the Internet) and "by the social distance of researchers and the quality and accessibility of research-relevant information" [6] (p. 392). (On this point, Melkers and Kiopa [6] cited, among others, Autant-Bernard, Billand, Frachisse, and Massard [46], who emphasized the impact of social distance or networks effect, as opposed to spatial distance or geographical effects, on individual researchers' cooperation behaviors.) We would point out, however, that inadequacies in the English language skills needed (e.g., those for composing emails, talking via Skype, participating in international conferences, or discussing research face-to-face with confidence) can greatly undercut the opportunity for communication to take place. Conversely, with the needed language skills, the problem of lacking access to potential collaborators can perhaps be overcome by a large measure, as CMAs could then take greater initiatives in seeking opportunities and establishing communication channels. Our CMA respondents placed getting help in English high on the list of benefits of entering partnership with OMAs, while citing difficulty in communication caused by language barriers and geographic distance as the greatest challenges. Our OMA respondents also pointed to CMAs' inadequacies in English proficiency and English writing ability. In our previous research, we have highlighted how inadequacies in English proficiency can become barriers for Chinese business/management scholars in their endeavors to publish in international journals, present papers at international conferences, and meet the institutional requirement of bilingual teaching [2,3,47]. In our view, the importance of English language skills has been underestimated in assessing international collaboration involving English-as-an-additional-language (EAL) scholars and their collaborators.

It is noteworthy that both CMA and OMA respondents in our study listed each other's good qualities as a plus for collaboration. OMAs were admired for their "intellectual passion, professionalism", and being "reliable", whereas CMAs were respected for their "upbeat morale, motivation to publish", and "diligence". It can be suggested that such mutual appreciation of good qualities potentially enhance trust, which is pivotal to successful research collaboration $[14,17,48]$. Yet, concerns over a lack of mutual understanding and trust may be more conspicuous for our CMA respondents. Notably, potential conflicts over authorship and responsibilities [49] were cited by both parties. Despite limited instances in our data, there were signs that the two parties had some reservations about each other's lack of participation. Specifically, some CMAs appeared to be worried about the practices of listing authors, whereas some OMAs seemed critical of their Chinese collaborators' understanding of the criteria for authorship and prioritizing "SSCI publication" over participation in research. The institutional force [24] has a stake here. We may suggest that the institutional pressure for CMAs to get published in international journals $[2,25,36]$ can greatly complicate issues of aims, trust, and contribution in international collaboration.

\section{Conclusions}

In this paper we reported a study of the perspectives taken by Chinese academics and their overseas counterparts on the benefits of engaging in research collaboration with each other and the challenges faced in the collaboration. The study revealed, at a more micro-level, some social dynamics of international collaboration, which have not been the focus of previous macro-level, bibliometric research on international collaboration. By examining international collaboration in a social science discipline, our study generates insights that complement the current discussions of the internationalization of social sciences [7,21]. Our focus on the discipline of management in an emerging economy would appeal to those interested in looking into comparable or different economic contexts, to examine the ecology of international collaboration and its implications. For those seeking to participate in collaboration with Chinese scholars, our study provides a valuable reference of the perspectives taken by (prospective) collaborative parties in one discipline, alerting them to the potential pluses and pitfalls lying ahead.

Our study also has implications for administrators and policy makers. Above all, efforts can be made, in particular, at institutional levels, to facilitate international collaboration [9]. These may 
include increasing financial support to expand the opportunities of academic exchanges, overseas stints during and after the doctoral study, and participating in international conferences held in China and overseas. In addition, there need to be more academic associations, such as IACMR and "boundary organizations" [10] (p. 367) to foster connections and communication between social scientists in periphery contexts and those in the center, and to redress the "asymmetrical structure of exchange" in social sciences in the long run [7] (p. 144). For EAL scholars, sustained provision of training in English language skills would be crucial. In many EAL contexts, traditional English teaching has focused on reading comprehension [3]; yet, the current demands of participating in knowledge construction at global levels have made English-medium communication skills for academic purposes indispensable. Massive resources need to be invested in the preparation of English for Academic Purposes (EAP) teachers and the set-up or strengthening of EAP programs in EAL contexts.

Our study has taken a preliminary step toward accessing the voices of individual academics to address an undertaking (i.e., international research collaboration) that characterizes the contemporary world of academia and yet remains inaccessible or challenging for many individual researchers [18]. Future research can take further insights from bibliometric research and be conducted on larger scales, for instance, by making cross-disciplinary and cross-institutional comparisons. The use of multiple methods will be necessary to reveal the complexities embedded in the process of cross-cultural research collaboration. There are also related issues to be addressed, such as differences in individual experiences, the roles played by the collaborating parties during research collaboration, and the learning curves of novices in participating in collaboration. Finally, it is hoped that our study will help to support reflexive practices amongst university academics, as we aim to engage in research collaborations with peers to carry forward our academic careers.

Acknowledgments: The study reported in this paper was part of a larger project on Chinese management academics' international publication endeavors, which was funded by the Faculty Research Fund at the Faculty of Education, University of Hong Kong. We would like to thank Meng Ge and Qianshan Chen for research assistance, and the two anonymous reviewers for their constructive feedback on an earlier version of the paper.

Author Contributions: The two authors jointly designed the study, and collected and analyzed the data. The first author drafted the paper and the two authors jointly revised the paper.

Conflicts of Interest: The authors declare no conflict of interest.

\section{Appendix A. Open-Ended Questions in the Two Questionnaires (Extracts from the Questionnaires)}

(1) Two Open-Ended Questions in Questionnaire One

\section{F. ADDITIONAL COMMENTS}

1. What are the primary benefits that you perceive of collaborating with academics outside Mainland China?

2. What difficulties/problems/disadvantages/challenges do you perceive of collaborating with academics outside Mainland China?

(2) Three Open-ended Questions in Questionnaire Two 
19. If there have been some changes in the pattern of your research collaboration with Mainland Chinese academics over the years, how would you describe such changes? (by reference to the statements in questions no. $17 \& 18$ above, if necessary)

\section{E. ADDITIONAL COMMENTS}

21. What are the primary benefits that you perceive of collaborating with Mainland Chinese academics?

22. What difficulties/problems/disadvantages/challenges do you perceive of collaborating with Mainland Chinese academics?

\section{References}

1. Zhai, L.; Yan, X.; Shibchurn, J.; Song, X. Evolutionary analysis of international collaboration network of Chinese scholars in management research. Scientometrics 2014, 98, 1435-1454. [CrossRef]

2. Li, Y. Seeking entry to the North American market: Chinese management academics publishing internationally. J. Engl. Acad. Purp. 2014, 13, 41-52. [CrossRef]

3. Li, Y.; Hu, G. Chinese management academics' English-medium scholarly experience: Comparative perspectives on overseas-trained and home-trained scholars. Ibérica 2017, 33, 71-96.

4. Tsui, A.S. Autonomy of inquiry: Shaping the future of emerging scientific communities. Manag. Organ. Rev. 2009, 5, 1-14. [CrossRef]

5. Zhou, P.; Glänzel, W. In-depth analysis on China's international cooperation in science. Scientometrics 2010, 82, 597-612. [CrossRef]

6. Melkers, J.; Kiopa, A. The social capital of global ties in science: The added value of international collaboration. Rev. Policy Res. 2010, 27, 389-414. [CrossRef]

7. UNESCO. World Social Science Report: Knowledge Divides; UNESCO and International Social Science Council: Paris, France, 2010.

8. Bozeman, B.; Corley, E. Scientists' collaboration strategies: Implications for scientific and technical human capital. Res. Policy 2004, 33, 599-616. [CrossRef]

9. González-Alcaide, G.; Gómez-Ferri, J. The tenets of scientific collaboration: Rules and recommendations. Curr. Sci. 2017, 113, 1505-1506.

10. Sá, C.M.; Oleksiyenko, A. Between the local and the global: Organized research units and international collaborations in the health sciences. High. Educ. 2011, 62, 367-382. [CrossRef]

11. van Rijnsoever, F.J.; Hessels, L.K.; Vandeberg, R.L.J. A resource-based view on the interactions of university researchers. Res. Policy 2008, 37, 1255-1266. [CrossRef]

12. Eisend, M.; Schmidt, S. The influence of knowledge-based resources and business scholars' internationalization strategies on research performance. Res. Policy 2014, 43, 48-59. [CrossRef]

13. Hara, N.; Solomon, P.; Kim, S.L.; Sonnenwald, D.H. An emerging view of scientific collaboration: Scientists' perspectives on collaboration and factors that impact collaboration. J. Am. Soc. Inf. Sci. Technol. 2003, 54, 952-965. [CrossRef]

14. Ou, A.Y.; Varriale, L.; Tsui, A.S. International collaboration for academic publication: Implications from the resource-based view and transaction cost theory. Group Organ. Manag. 2012, 37, 407-451. [CrossRef]

15. Abramo, G.; D'Angelo, C.A.; Solazzi, M. The relationship between scientists' research performance and the degree of internationalization of their research. Scientometrics 2011, 86, 629-643. [CrossRef]

16. Gilbert, N. Research sans frontières. Nature 2011, 471, 559. [CrossRef] [PubMed]

17. Easterby-Smith, M.; Malina, D. Cross-cultural collaborative research: Toward reflexivity. Acad. Manag. J. 1999, 42, 76-86. [CrossRef] 
18. Hoffman, D.M.; Blasi, B.; Ćulum, B.; Dragšić, Ž.; Ewen, A.; Horta, H.; Nokkala, T.; Rios-Aguilar, C. The methodological illumination of a blind spot: Information and communication technology and international research team dynamics in a higher education research program. High. Educ. 2014, 67, 473-495. [CrossRef]

19. Thomas, R.; Tienari, J.; Davies, A.; Meriläinen, S. Let's talk about "us": A reflexive account of a cross-cultural research collaboration. J. Manag. Inq. 2009, 18, 313-324. [CrossRef]

20. Zhou, P.; Thijs, B.; Glänzel, W. Is China also becoming a giant in social sciences? Scientometrics 2009, 79, 593-621. [CrossRef]

21. Brew, A.; Boud, D.; Lucas, L.; Crawford, K. Reflexive deliberation in international research collaboration: Minimising risk and maximizing opportunity. High. Educ. 2013, 66, 93-104. [CrossRef]

22. Hyland, K. Academic Publishing: Issues and Challenges in the Construction of Knowledge; Oxford University Press: Oxford, UK, 2015.

23. Aydinli, E.; Mathews, J. Are the core and periphery irreconcilable? The curious world of publishing in contemporary international relations. Int. Stud. Perspect. 2000, 1, 289-303. [CrossRef]

24. Peterson, M.F. International collaboration in organizational behavior research. J. Organ. Behav. 2001, 22, 59-81. [CrossRef]

25. Shao, J.; Shen, H. The outflow of academic papers from China: Why is it happening and can it be stemmed? Learn. Publ. 2011, 24, 95-97.

26. Morgan, J. China on the Fast Track. Times High. Educ. 2013. Available online: http://www. timeshighereducation.co.uk/features/china-on-the-fast-track/2009923.article (accessed on 1 August 2015).

27. Yang, R.; Xie, M. Learning toward the centers: International networking at China's five C9 League universities. Front. Educ. China 2015, 10, 66-90.

28. Deng, Z. Westernization of the Chinese social sciences: The case of legal science (1978-2008). In World Social Science Report: Knowledge Divides; UNESCO \& International Social Science Council: Paris, France, 2010; pp. 182-185.

29. Yang, R. Internationalisation; indigenisation and educational research in China. Aust. J. Educ. 2005, 49, 66-88. [CrossRef]

30. Guo, Y. Difangxing zhishi: Tongwang xueshu zizhuxing de ziyou zhi lu—“Guanlixue zai zhongguo” zhi wo jian [Local knowledge: A free path to academic autonomy-My views on "Management in China". Guanli Xuebao [Chin. J. Manag.] 2010, 7, 475-488.

31. Barney, J.B.; Zhang, S. The future of Chinese management research: A theory of Chinese management versus a Chinese theory of management. Manag. Organ. Rev. 2009, 5, 15-28. [CrossRef]

32. Tsui, A.S. From homogenization to pluralism: International management research in the academy and beyond. Acad. Manag. J. 2007, 50, 1353-1364. [CrossRef]

33. Lave, J.; Wenger, E. Situated Learning: Legitimate Peripheral Participation; Cambridge University Press: Cambridge, UK, 1991.

34. Merriam, S.B. Qualitative Research: A Guide to Design and Implementation; Jossey-Bass: San Francisco, CA, USA, 2009.

35. Saldaña, J. The Coding Manual for Qualitative Researchers; Sage: Thousand Oaks, CA, USA, 2009.

36. Li, Y.; Yang, R. Chinese business schools pursuing growth through international publishing: Evidence from institutional genres. In Global Academic Publishing: Policies, Perspectives, and Pedagogies; Curry, M.J., Lillis, T., Eds.; Multilingual Matters: Bristol, UK, 2017; pp. 50-69.

37. Alatas, S.F. Academic dependency and the global division of labour in the social sciences. Curr. Sociol. 2003, 51, 599-613. [CrossRef]

38. Zhang, H.; Cong, Y. China. In Handbook of Global Bioethics; ten Have, H.A.M.J., Gordijn, B., Eds.; Springer: Dordrecht, The Netherlands, 2014; pp. 993-1009.

39. Qiu, J. Publish or perish in China. Nature 2010, 463, 142-143. [CrossRef] [PubMed]

40. Whetten, D.A. An examination of the interface between context and theory applied to the study of Chinese organizations. Manag. Organ. Rev. 2009, 5, 29-55. [CrossRef]

41. Melin, G. Pragmatism and self-organization: Research collaboration on the individual level. Res. Policy 2000, 29, 31-40. [CrossRef]

42. Shi, L. Writing in two cultures: Chinese professors return from the West. Can. Mod. Lang. Rev. 2003, 59, 369-391. [CrossRef] 
43. Adler, N.J.; Campbell, N.; Laurent, A. In search of appropriate methodology: From outside the People's Republic of China looking in. J. Int. Bus. Stud. 1989, 20, 61-74. [CrossRef]

44. Tsui, A. Contributing to global management knowledge: A case for high quality indigenous research. Asia Pac. J. Manag. 2004, 21, 491-513. [CrossRef]

45. Boyacigiller, N.A.; Adler, N.J. The parochial dinosaur: Organization science in a global context. Acad. Manag. Rev. 1991, 16, 262-390.

46. Autant-Bernard, C.; Billand, P.; Frachisse, D.; Massard, N. Social distance versus spatial distance in R\&D cooperation: Empirical evidence from European collaboration choices in micro and nanotechnologies. Pap. Reg. Sci. 2007, 86, 495-519.

47. Hu, G.; Lei, J. English-medium instruction in Chinese higher education: A case study. High. Educ. 2014, 67, 551-567. [CrossRef]

48. Shrum, W.; Chompalov, I.; Genuth, J. Trust, conflict and performance in scientific collaborations. Soc. Stud. Sci. 2001, 31, 681-730. [CrossRef]

49. Bozeman, B.; Yutie, J. Trouble in paradise: Problems in academic research co-authoring. Sci. Eng. Ethics 2016, 22, 1717-1743. [CrossRef] [PubMed]

(C) 2018 by the authors. Licensee MDPI, Basel, Switzerland. This article is an open access article distributed under the terms and conditions of the Creative Commons Attribution (CC BY) license (http:/ / creativecommons.org/licenses/by/4.0/). 